\title{
Effets d'un stress hydrique appliqué à différents stades de développement sur l'aspect quantitatif et qualitatif des semences chez un écotype autochtone de sorgho grain (Sorghum bicolor)
}

Leila Radhouane, Nesrine Aissa et Leila Romdhane

Institut National de la Recherche Agronomique de Tunis, Avenue Hédi Karray-2049 Ariana- Tunisie

Auteur correspondant : radhouane.leila@iresa.agrinet.tn

Original submitted in on $15^{\text {th }}$ January 2014 Published online at www.m.elewa.org on 28 th February 2014. http://dx.doi.org/10.4314/jab.v74i1.6

\section{RÉSUMÉ}

Objectif : Les effets d'un stress hydrique appliqué à différents stades de développement sur l'aspect quantitatif et qualitatif des semences ont été étudiés chez un écotype autochtone de sorgho grain.

Méthodologie et résultats : L'essai a été conduit en pots de végétation. L'irrigation des plantes a été faite par pesées successives des pots. Au cours de chaque pesée, le témoin est ramené au même poids correspondant à $70 \%$ de la réserve en eau utile ; alors que le traitement stressé (SI, S2 et S3) maintient le contenu en eau à $30 \%$ de la RUE pendant 10 jours. A la fin du cycle de manque d'eau, l'irrigation a été reprise comme pour le témoin. Les stades concernés par le stress hydrique sont la phase d'initiation florale (S1), la phase de gonflement-épiaison (S2) et la phase de floraison - remplissage des grains (S3). Les résultats ont montré que le rendement et la qualité des semences baissent significativement lorsque le stress est appliqué au stade $S 2$ et que le taux de germination et la vigueur des plantules (issues de S2) baissent en conséquence.

Conclusion et application : de tels résultats amènent à affirmer que la phase phénologique la plus sensible au stress hydrique est celle du gonflement-épiaison. L'importance de cette étude trouve son application dans la gouvernance de l'irrigation. En effet, dans des conditions de pénurie d'eau, il faut mettre en place des scénarii permettant de livrer l'eau aux plantes seulement aux stades critiques de leur croissance afin d'éviter tout gaspillage.

Mots clés : Sorgho, Écotype autochtone, Stress hydrique, Phase de développement, Rendement en grains

\begin{abstract}
:
Effects of water stress applied at different stages of development on the quantity and quality of autochthonous sorghum seed ecotype (Sorghum bicolor)

Objective: The effects of water stress applied at different stages of development on the quantity and quality of seeds were studied in native grain sorghum ecotype.

Methodology and Results: The trial was conducted in plant pots. The irrigation of the plants was made together with the successive weighing pots. During each weighing, the control was returned to the same weight corresponding to $70 \%$ of the UWR (useful water reserve), whereas stress treatment (SI, S2 and S3) maintained the water content to $30 \%$ of the UWR for 10 days. At the end of the cycle of water shortage, irrigation was taken
\end{abstract}




\section{Radhouane et al. J. Appl. Biosci. 2014. Effets d'un stress hydrique applique a différents stades de développement des semences chez un écotype autochtone de sorgho grain}

as the control. Stages affected by water stress were the floral initiation stage (S1), the swelling phase-heading (S2) and the flowering phase - grain filling (S3). The results showed that the yield and seed quality drop significantly when stress is applied at S2 and that the rate of germination and seedling vigour (seeds from S2) fall accordingly.

Conclusion and application: these results lead to affirm that the most sensitive water stress phase is that of swelling-heading. The importance of this study is its application in the governance of irrigation. Indeed, under conditions of water scarcity, we must develop t scenarios to deliver water to plants only at critical stages of growth in order to avoid waste.

Key words: Sorghum, Autochthonous ecotype, Water stress, Phase development, Grain yield.

\section{INTRODUCTION}

La rareté des ressources en eau autour de la Méditerranéen, leur fragilité et leur inégale répartition font naître un risque majeur de pénurie, qui en dépit de toutes les tentatives pour accroître l'offre semble inéluctable (Benblidia et al., 1998). Malgré des siècles d'efforts de maîtrise consacrés à la réalisation d'aménagements visant à améliorer la disponibilité de l'eau, cette dernière demeure une limite fondamentale pour le développement des activités économiques, notamment agricoles, dans les pays du Sud du bassin méditerranéen. L'augmentation importante des pressions humaines sur les ressources depuis quelques décennies se traduit par des situations de concurrence très nettes entre secteurs d'utilisation. Quoique les cultures irriguées bénéficient encore le plus souvent de ressource en eau suffisante pour couvrir leurs besoins en eau, la situation a changé depuis quelques années car le contexte hydraulique a évolué et le nombre de petites régions concernées par des ressources en eau limitées a augmenté. Dans ces conditions de pénurie d'eau, il faut mettre en place des scénarii conduisant à une stabilisation de la pression sur les ressources en eau qui sont basés sur la mise en place de nouvelles politiques de l'eau qui consistent à mieux prendre en compte le processus de raréfaction des ressources et à mettre l'accent sur la nécessité d'une meilleure gouvernance. Ceci implique notamment que l'eau ne doit être livrée aux cultures qu'aux stades critiques de leur croissance afin d'éviter tout gaspillage. De ce fait, il est important de reconnaître ces étapes critiques de la croissance pour toutes les cultures (Seghatoleslam et al., 2008). Dans ce contexte, la phase d'apport aurait un effet plus important que la quantité d'eau apportée (Radhouane et Ben Salem,
1998 ; Tuinstra et al., 1997). Le déficit en eau est l'une des contraintes les plus courantes de l'environnement qui influe sur la croissance et le développement des plantes (Sadras and Milroy, 1996 ; Aslam et al., 2006). Cette sécheresse ou manque d'eau qui peut intervenir à différentes périodes du cycle de la vie de la plante est étroitement lié au stade de développement de la plante (Chaves et al., 2002; Jaleel et al., 2008) et peut affecter le rendement en biomasse et en grains des cultures (Araus et al., 2002; Tester and Bacic, 2005). En effet, plusieurs études ont montré que, lors d'un déficit hydrique, les plantes adoptent des stratégies d'adaptation qui diffèrent d'une espèce à une autre et qui font intervenir une large combinaison de facteurs morphologiques, physiologiques et biochimiques (Farooq et al., 2008; Reynolds and Tuberosa, 2008; Razmjoo et al., 2008). L'évaluation de l'effet d'un déficit hydrique sur les composantes du rendement aussi bien sur le plan qualitatif que quantitatif, en relation avec les différents stades de développement de la plante a été étudié chez le sorgho grain. Le choix de cette culture est justifié tout d'abord par l'aspect adaptatif de l'écotype aux conditions locales, et aussi par ses avantages aussi bien nutritifs qu'économiques. En effet, le sorgho est une céréale qui possède des valeurs nutritives plus importantes en protéines que certains aliments de base utilisés par les éleveurs tunisiens. De plus, face aux hausses continues des prix de l'aliment concentré sur le marché mondial, dont l'impact au niveau des coûts d'exploitation des élevages en Tunisie, n'est plus à démontrer, il devient donc inévitable de soutenir tous ceux qui présentent des idées qui s'avèrent économiquement plus rentables. De ce fait, le sorgho apparaît comme 


\section{Radhouane et al. J. Appl. Biosci. 2014. Effets d'un stress hydrique applique a différents stades de développement des semences chez un écotype autochtone de sorgho grain}

une culture attractive, mais dont le développement est pénalisé par le niveau bas et la variabilité des rendements. Le développement de la production de cette plante fourragère devrait permettre de faire face à la flambée des prix de l'orge, du maïs et du soja qui est de nature à affecter l'élevage des ovins, bovins et des volailles. L'objectif de ce travail est de tester cet écotype autochtone de sorgho (famille des Poaceae) dans des conditions de stress hydriques lors de différentes phases de développement et de croissance afin de l'inclure dans un programme de sélection de génotypes répondant à un objectif de haut rendement en conditions de sécheresse.

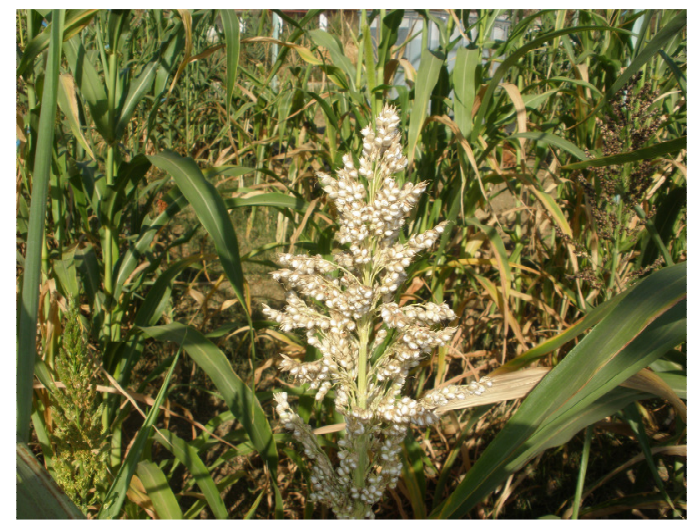

Sorgho en plein champs

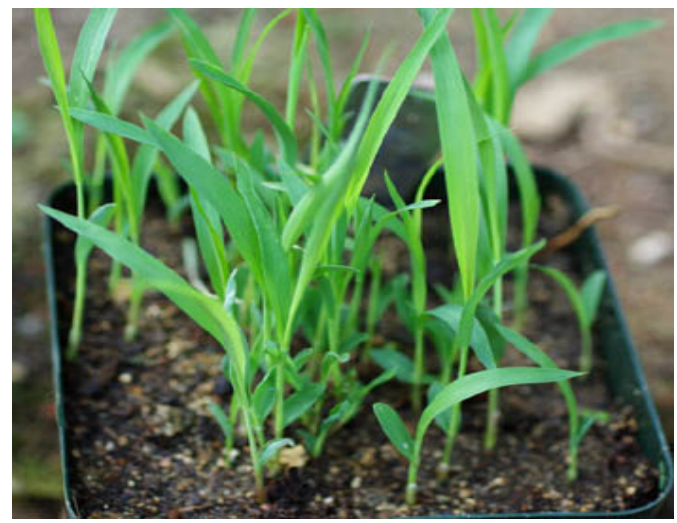

Sorgho dans des pots

\section{MATÉRIEL ET MÉTHODES}

Matériel végétal : L'étude a porté sur un écotype de sorgho grain (Sorghum bicolor) autochtone ramassé dans la région de Fernana (Nord Ouest de la Tunisie). II présente une taille de 2 à $3 \mathrm{~m}$ et possède une panicule relativement lâche et érigée à grains blancs. Le poids de 1000 grains est de $20-25 \mathrm{~g}$.

Au cours de ces dernières années, les surfaces qui lui sont consacrées ont connu une forte régression.

Conduite de l'essai : L'essai a été conduit en pots de végétation qui peuvent contenir jusqu'à $25 \mathrm{~kg}$ de terre. Chaque pot est rempli de $20 \mathrm{~kg}$ de terre à $6 \%$ d'humidité, soit $18,8 \mathrm{~kg}$ de matière sèche. Le semis a été effectué à raison de par pot. Une fertilisation azotée a été apportée en 2 temps: la moitié au semis et l'autre moitié au tallage. La dose étant calculée sur la base de $100 \mathrm{~kg} / \mathrm{ha}$ d'ammonitrate à $33 \%$. Le milieu de culture est formé par une terre argileuse prélevée sur la couche arable de la station de I'INRAT à I'Ariana. Les caractéristiques moyennes de la courbe $\mathrm{pF}$ de ce sol sont les suivantes: - pF 4,2 = 18 (point de flétrissement permanent) - $\mathrm{pF} 2,7=25$ (capacité au champ)

L'irrigation des plantes a été faite par pesées successives des pots. Au cours de chaque pesée, le témoin est ramené au même poids correspondant à $70 \%$ de la RUE; alors que le traitement stressé (SI, S2 et S3) maintient le contenu en eau à $30 \%$ de la RUE. La durée de chaque stress est de 10 jours. A la fin du cycle de manque d'eau, l'irrigation a été reprise comme pour le témoin. Les irrigations ont été réalisées à une périodicité de 3 jours. Les apports complètent la masse d'eau au seuil du traitement $(70 \%$ ou $30 \%)$.

Traitements : Une même durée de stress (10 jours avec $30 \%$ de la RUE) a été imposée au cours de chaque phase de développement ciblée par le stress, soit :

- stress appliqué en phase d'initiation florale (S1) soit 29 jours après semis (jas)

- stress en phase de gonflement-épiaison (S2) soit 73ème jas

- stress en phase de floraison - remplissage des grains (S3) appliqué au 91ème jas.

Mesures réalisées : Les mesures d'aspect quantitatif ont concerné le rendement en paille et le rendement en grains et ses composantes. Les paramètres étudiés sont :

- Poids sec de la biomasse aérienne (MSA) en $g$

- Poids sec des panicules (PSP) en g

- Nombre de grains par pied (NGP)

- Poids des grains par pieds (PGP) en g

- Poids de 100 gains (P100G) en g

Toutes les pesées ont été réalisées à l'aide d'une balance de précision à $0.1 \mathrm{~g}$. Pour ce qui est de l'appréciation de l'aspect qualitatif des semences, il a été procédé à la mesure du poids individuel des grains, à la détermination 


\section{Radhouane et al. J. Appl. Biosci. 2014. Effets d'un stress hydrique applique a différents stades de développement des semences chez un écotype autochtone de sorgho grain}

de leur taille et aussi à un test de germination des semences.

Poids et mesure individuels des grains : Un lot de 100 grains par répétition est prélevé pour chaque traitement. Leur poids individuel est déterminé à l'aide d'une balance de précision Mettler à 0,001 $\mathrm{g}$. Les grains du même lot sont mesurés à l'aide d'un pied à coulisse (précision $0.001 \mathrm{~mm})$.

Test de germination : Les grains issus des différents traitements (50 grains/répétition) sont mis dans des boîtes de Pétri ayant comme substrat du papier filtre. Ces boîtes sont placées dans une étuve où la température est

\section{RÉSULTATS}

Effet du stress hydrique sur le rendement et ses composantes (Aspect quantitatif): Les processus impliqués dans l'élaboration du rendement d'une culture sont influencés par deux types de facteurs, les facteurs génétiques (intrinsèques à la plante) et les facteurs environnementaux. Les interactions génotypes-milieu jouent également un rôle important. Parmi les caractéristiques morphologiques qui interviennent dans l'élaboration du rendement, nous citons la production de la biomasse et les composantes du rendement (comme le poids sec des panicules, le nombre et le poids de grains fixée à $25^{\circ} \mathrm{C}$. Un comptage de plantules normales est effectué au bout d'une semaine. Le pourcentage de germination est calculé pour chaque répétition et la longueur des parties aérienne et racinaire des plantules est mesurée.

Étude statistique : L'essai a été du type complètement randomisé comportant 4 traitements et 16 répétitions. L'analyse de la variance a été faite par le logiciel STATITCF, chaque donnée constitue la moyenne de 5 mesures. Pour la comparaison des moyennes, le test de Bonferroni au seuil de $5 \%$ a été appliqué.

par pied et le poids de 100 gains). A la lumière des résultats présentés dans le tableau 1 , nous constatons que les différents traitements ont produit la même quantité de matière sèche aérienne que le témoin. Pour les autres composantes de rendement, c'est le traitement S2 qui a présenté les valeurs significativement les plus faibles par rapport aux autres traitements et par rapport au témoin. En effet, le stress $S 2$ appliqué durant la phase de gonflement, a réduit le PGP, le PSP, le P100G et le NGP respectivement de $60 \%, 50 \%, 35 \%$ et $52 \%$.

Tableau 1 : Effet de l'application de stress hydrique à différents stades de développement sur le rendement enmatière sèche et sur les composantes du rendement

\begin{tabular}{|c|c|c|c|c|}
\hline Paramètre & Témoin (T) & Stressé 1 (S1) & Stressé 2 (S2) & Stressé 3 (S3) \\
\hline MSA (g) & $364.5 \mathrm{a}$ & $360.9 \mathrm{a}$ & $358.2 \mathrm{a}$ & $334.8 \mathrm{a}$ \\
PGP $\mathbf{( g )}$ & $14.6 \mathrm{a}$ & $12.2 \mathrm{a}$ & $5.8 \mathrm{~b}$ & $11.1 \mathrm{a}$ \\
PSP $\mathbf{g})$ & $47.3 \mathrm{a}$ & $43.8 \mathrm{a}$ & $23.8 \mathrm{~b}$ & $40.0 \mathrm{a}$ \\
P100G $\mathbf{( g )}$ & $2.0 \mathrm{a}$ & $1.8 \mathrm{a}$ & $1.3 \mathrm{~b}$ & $1.7 \mathrm{ab}$ \\
NGP & $661 \mathrm{a}$ & $558 \mathrm{ab}$ & $316 \mathrm{c}$ & $476 \mathrm{bc}$ \\
\hline
\end{tabular}

Les chiffres de la même ligne suivis d'une même lettre ne sont pas significativement différents (alpha $=0.05)$

$\mathrm{MSA}=$ poids sec de la biomasse aérienne en $\mathrm{g} ; \mathrm{PGP}=$ poids des grains par pieds en $\mathrm{g} ; \mathrm{PSP}=$ poids sec des panicules en $\mathrm{g}$;

$\mathrm{P} 100 \mathrm{G}=$ poids de 100 grains en $\mathrm{g} ; \mathrm{NGP}=$ nombre de grains par pied

Effet du stress hydrique sur la qualité des grains (Aspect qualitatif)

Distribution des grains de sorgho selon leur taille (en $\%$ d'un lot de 100 grains): L'effet d'une contrainte hydrique sur la distribution des grains selon leur taille dans un lot de 100 grains a montré que les traitements
S1 et S2 présentent un pourcentage plus élevé de grains de taille inférieure à $2,5 \mathrm{~mm}$ par rapport au témoin et à S3 (Figure 1). Le pourcentage cumulé de grains dont la taille est inférieure à 3,5 mm est le plus élevé pour S3 (82\%), suivi de S1 $(77 \%)$. Le témoin et S2 montrent le pourcentage le plus faible $(69 \%)$. 

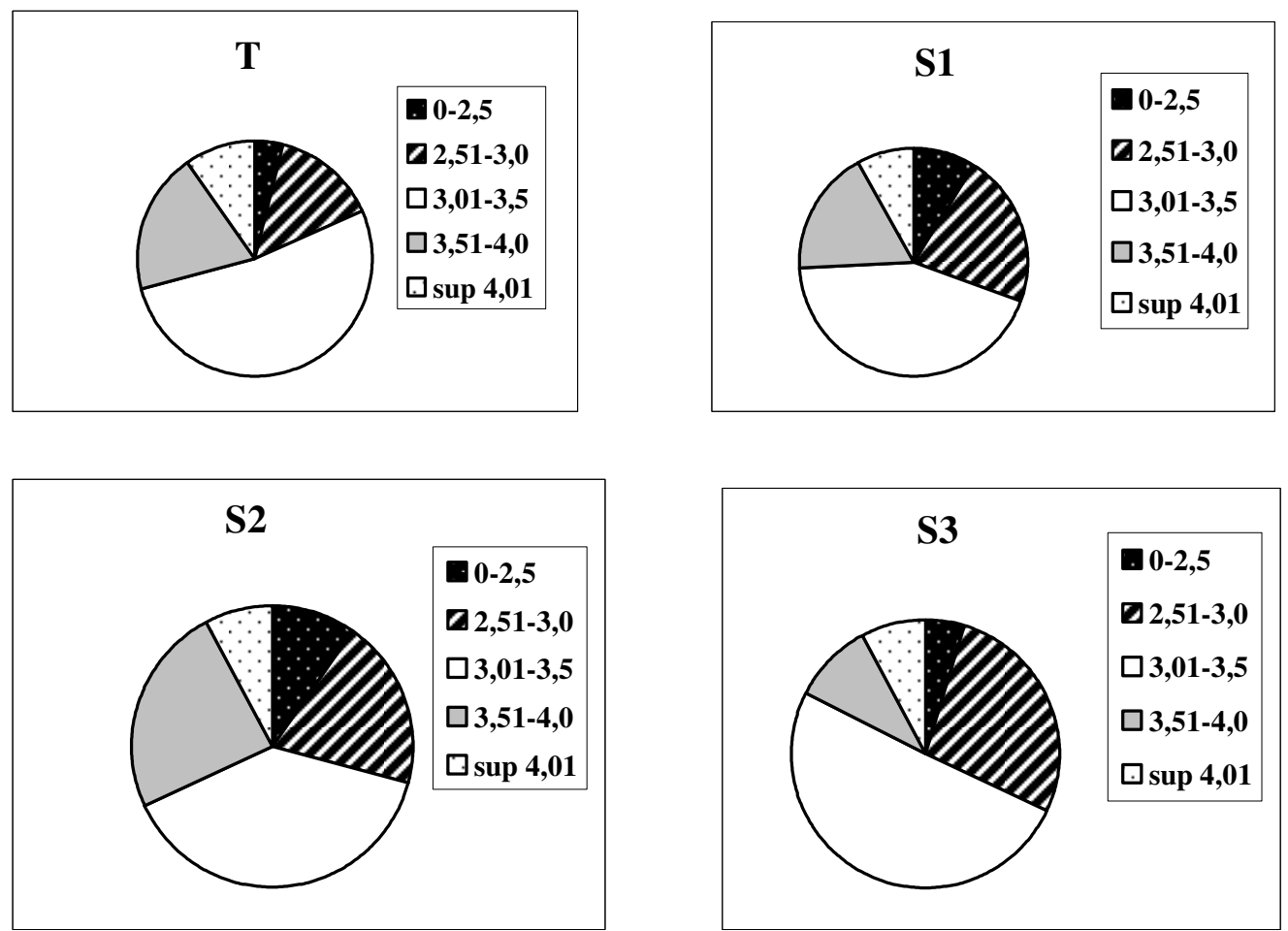

Figure 1. Distribution des grains de sorgho selon leur taille $(\mathrm{mm})$ dans un lot de 100 grains; $T=$ témoin, $\mathrm{S} 1=$ stress appliqué en phase d'initiation florale, S2= stress appliqué en phase de gonflement, S3= stress en phase de floraison - remplissage des grains

Distribution des grains de sorgho selon le poids individuel d'un grain : Le régime hydrique a eu un effet significatif sur le poids individuel des grains (figure 2) : le témoin et $\mathrm{S} 1$ ont donné en moyenne le poids individuel de grain le plus élevé (environ $22 \mathrm{mg}$ ). Ils sont suivis de S3 $(19 \mathrm{mg})$ et de S2 $(16 \mathrm{mg})$. Le traitement S2 a également donné le nombre le plus élevé en grains de faible poids (entre 4-9 mg et 9.1-14 mg). Pour les intervalles de poids compris entre 14,1 et $19 \mathrm{mg}$ et 19,1 et $24 \mathrm{mg}$. Ce même traitement S2 a vu le poids de ses grains réduit respectivement de $30 \%$ et de $76 \%$ par rapport au témoin. Cette diminution atteint $38 \%$ et $72 \%$ par rapport à S3.
Germination et développement des plantules issues de semences stressées: Les semences issues de plantes stressées à différents stades de développement ont montré des taux de germination différents. En effet, la germination la plus faible est donnée par le traitement $\mathrm{S} 2$. Les traitements S1 et S3 ne sont pas significativement différents entre eux et aussi avec le témoin (tableau 2). Pour ce qui est du développement de la plantule, c'est le traitement S1 qui a donné la plantule la plus vigoureuse avec une longueur totale et une longueur des parties aériennes et racinaire significativement supérieure à celle du témoin. 


\section{Radhouane et al. J. Appl. Biosci. 2014. Effets d'un stress hydrique applique a différents stades de développement des semences chez un écotype autochtone de sorgho grain}

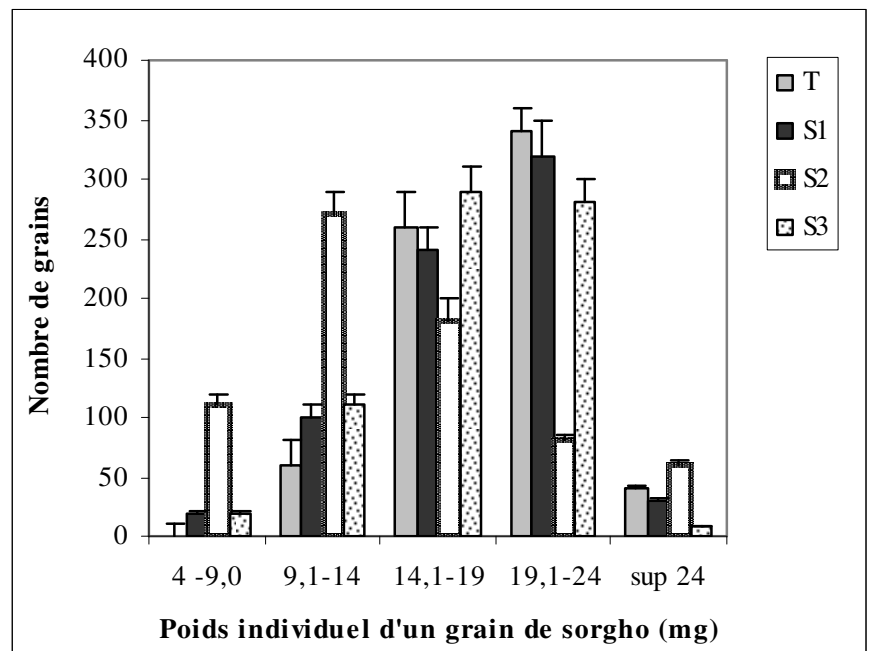

Figure 2. Distribution des grains de sorgho selon le poids individuel d'un grain (mg)

Bien que son taux de germination n'ait pas été réduit par l'application du stress hydrique, le traitement S3 a réduit le développement de la plantule. Comme pour la germination, c'est S2 qui a généré la plantule la moins vigoureuse.

Tableau 2 : Taux de germination et longueur du coléoptile, de la radicule et de la plantule des semences issues des différents traitements

\begin{tabular}{|c|c|c|c|c|}
\hline Paramètre & Témoin $\mathbf{( T )}$ & Stressé 1(S1) & Stressé 2 (S2) & Stressé 3 (S3) \\
\hline $\mathbf{G}(\%)$ & $82.0 \mathrm{a}$ & $76.0 \mathrm{a}$ & $60.0 \mathrm{~b}$ & $77.0 \mathrm{a}$ \\
LR $(\mathbf{c m})$ & $5.5 \mathrm{~b}$ & $10.4 \mathrm{a}$ & $4.2 \mathrm{~b}$ & $5.3 \mathrm{~b}$ \\
LC $(\mathbf{c m})$ & $13.0 \mathrm{~b}$ & $18.0 \mathrm{a}$ & $6.2 \mathrm{C}$ & $7.3 \mathrm{C}$ \\
LT $(\mathbf{c m})$ & $18.5 \mathrm{~b}$ & $28.4 \mathrm{a}$ & $10.4 \mathrm{C}$ & $12.6 \mathrm{C}$ \\
\hline
\end{tabular}

Les chiffres de la même ligne suivis d'une même lettre ne sont pas significativement différents (alpha $=0.05$ ) ;G $=$ taux de germination en \%; LR= longueur de la radicule en $\mathrm{cm} ; \mathrm{LC}=$ longueur du coléoptile en $\mathrm{cm} ; \mathrm{LT}=$ longueur totale de la plantule en $\mathrm{cm}$

\section{DISCUSSION}

Le comportement aussi bien morphologique que physiologique d'une plante donnée dépend de l'espèce ou de la variété, de la durée et de la sévérité de la sécheresse ainsi que du moment de son application (Chaves et al., 2002). Au cours de cette expérimentation, il a été montré que la contrainte hydrique a agi différemment sur le sorgho aussi bien sur le plan quantitatif que qualitatif et ceci en fonction du stade de développement de la plante. Pour la production de la matière sèche aérienne, le moment d'imposition de la contrainte n'a pas eu d'effet significatif. En effet, tous les traitements ont donné statistiquement le même résultat. II semble que cet écotype de sorgho soit doté de mécanismes physiologiques de rattrapage de la croissance végétative après un stress hydrique. Dans ce cadre, Morizet et al. (1984) ont montré que les plantes résistantes à la sécheresse sont capables de produire une quantité de matière sèche après réhydratation, comparées aux plantes témoins. Un stress hydrique appliqué au début de la phase gonflement-épiaison ou au début de la phase floraison-remplissage des grains n'a pas d'effets significatifs limitant la production de matière sèche végétale. Dans des situations contraignantes où le manque d'eau ne dépasse pas une durée de 10 jours, la capacité photosynthétique n'est pas affectée, le rendement quantique reste efficace et des valeurs proches du témoin sont enregistrées juste après la réhydratation (Lal and Edwards, 1996; Saccardy et al., 1996; Foyer et al., 1998) . L'effet du stress hydrique sur les composantes de rendement a montré que c'est le traitement S2 qui a présenté les valeurs significativement les plus faibles par rapport aux autres traitements et par rapport au témoin. En effet, le stress S2 appliqué durant la phase de gonflement, a réduit le PGP, le PSP, le P100G et le NGP respectivement de $60 \%, 50 \%, 35 \%$ et $52 \%$. Cette baisse de rendement serait due à la 


\section{Radhouane et al. J. Appl. Biosci. 2014. Effets d'un stress hydrique applique a différents stades de développement des semences chez un écotype autochtone de sorgho grain}

diminution de la surface évapotranspirante par la sénescence des feuilles. II s'ensuit une chute de l'activité photosynthétique (Dwivedi et al., 2008) et une diminution de la quantité de photosynthétats. Le processus de remplissage des grains est alors ralenti voire bloqué. Des avortements de panicules entières ont été observés (Araus et al., 2011). Par contre, le traitement S1 a donné des résultats similaires au témoin. II semble qu'un déficit hydrique précoce et momentané n'a pas de conséquence notable sur les composantes de rendement. $\mathrm{Ce}$ phénomène serait du d'après certains auteurs (Araus et al., 1989), au contrôle stomatique qui est plus efficace aux stades jeunes. En effet, la plante réagit rapidement à toute déficience en eau en fermant ses stomates et dès que les conditions deviennent non contraignantes, la plante est susceptible de reprendre sa croissance et de compenser les pertes enregistrées durant les périodes déficientes en eau. Sur le plan qualitatif, il a été remarqué que l'application d'une contrainte hydrique au stade gonflement (S2) a donné le pourcentage le plus élevé en grains de petite taille et aussi le nombre le plus important en en grains de faible poids (entre 4-9 mg et 9.1-14 mg). De même, les graines issues du traitement $\mathrm{S} 2$ ont en moyenne un poids individuel très faible. Un tel résultat a été signalé par Prabhudeva et al. (1998) qui ont montré qu'un déficit hydrique survenu au stade gonflementépiaison est plus désastreux sur la qualité des grains que celui appliqué au stade floraison - remplissage des

\section{CONCLUSION}

Dans ces conditions de pénurie d'eau, il faut mettre en place des scénarios conduisant à une meilleure gouvernance de l'irrigation. Parmi les scénarios proposés, celui de livrer l'eau aux plantes seulement aux stades critiques de leur croissance afin d'éviter tout gaspillage. L'effet d'un déficit hydrique sur les composantes du

\section{RÉFÉRENCES BIBLIOGRAPHIQUES}

Araus, J.L., Tapia, L. and Alegre, L.; 1989. The effect of changing sowing date on leaf structure and gas exchange characteristics of wheat flag leaves grown under Mediterranean climate conditions. J. Exp.Bot., 40(215) : 639-646

Araus, J.L., Slafer, G.A., Reynolds, M.P. and Royo, C. ; 2002. Plant breeding and drought in $\mathrm{C} 3$ cereals : what should we breed for? Ann. Bot. 89, (Spec. No.) : 925-940.

Araus, J.L. ; Slafer, J.A. ; Royo, C. and Serret, M.D. ; 2011. Breeding for Yield Potential and Stress Adaptation in Cereals. Critical Reviews in Plant Science, 27: 377-412 grains. De même, Edward and Wright (2008) ont trouvé que les grains de blé issus de plantes stressées au stade pré anthèse sont ratatinés et de petites tailles. Ce même résultat est corroboré par Monneveux et al. (2006) sur le maïs. La connaissance du poids de la semence d'origine, qui détermine la capacité de résistance du végétal à des conditions adverses, constitue un élément essentiel de la caractérisation d'une plantule. En effet, les plantules issues de petites semences montreront une plus grande fragilité vis-à-vis de conditions d'environnement défavorables (Derieux et al., 1989). Pour cette raison, les caractères liés à la taille des semences sont importants à considérer en agronomie (Siddiq et al ., 2007). En effet, des semences de bonne qualité donnent une émergence rapide et uniforme qui à son tour assurera un haut rendement aussi bien sur le plan quantitatif que qualitatif (Yari et al ., 2010).D'après nos essais, les semences issues du traitement S2 ont donné le taux de germination le plus faible et ont généré les plantules les moins vigoureuses. On peut en conclure qu'un stress appliqué pendant la phase gonflement-épiaison affecte plus l'aspect qualitatif des semences que lorsqu'il est appliqué à la phase floraison - remplissage des grains. Un tel résultat nous permet de conclure que le choix des semences est un critère important dont on doit tenir compte si nous voulons avoir des rendements uniformes et importants.

rendement aussi bien sur le plan qualitatif que quantitatif, en relation avec les différents stades de développement de la plante a été étudié chez un écotype autochtone de sorgho grain a montré que le stade le plus sensible au manque d'eau est la phase gonflement-épiaison.

Aslam, M., Khan, I. A., Saleem, M. and Ali, Z. ; 2006. Assessment of water stress tolerance in different maize accessions at germination and early growth stage. Pak. J. Bot., 38(5) : 1571- 1579.

Benblidia, M., Margat, J. et Vallée, D. ; 1998. Pénuries d'eau prochaines en Méditerranée ? Futuribles, 233: 5-29.

Chaves, M.M.; Pereira, J.S.; Maroco, J.; Rodriques, M.L.; Ricardo, C.P.P.; Osorio, M.L. Carvatho, I.; Faria, T. and Pinheiro, C.; 2002. How plants cope with water stress in the field photosynthesis and growth ? Ann. Bot., 89: 907916 


\section{Radhouane et al. J. Appl. Biosci. 2014. Effets d'un stress hydrique applique a différents stades de développement des semences chez un écotype autochtone de sorgho grain}

Derieux, M. ; Bourdu, R. ; Duburcq, J.B. ; Boizard, H. ; 1989. La crise de croissance de la plantule de maïs à basse température. Agronomie, 9: 207212

Dwivedi, S. ; Perotti, E. and Ortiz, R. 2008. Towards molecular breeding of reproductive traits in cereal crops. Plant Biotechnol. J., 6: 529-559.

Edward, D. and Wright, D. ; 2008. The effects of winter water-logging and summer drought on the growth and yield of winter wheat (Triticum aestivum L.) European J. Agron., 28: 234-244

Farooq, M., Basra, S.M.A., Wahid, A., Cheema, Z.A., Cheeman M.A. and Khaliq, A. ; 2008. Physiological role of exogenously applied glycinebetaine in improving drought tolerance of fine grain aromatic rice (Oryza sativa L.). J. Agron. Crop Sci., 194: 325-333

Foyer, C.H. ; Valadier, M.H. ; Migge, A. and Becker, T.W. ; 1998. Drought-induced effects on nitrate reductase activity and mRNA and on the coordination of nitrogen and carbon metabolism in maize leaves. Plant Physiology, 117: 283292.

Jaleel, C.A. ; Gopi, R. and Panneerselvam, R. ; 2008. Growth and photosynthetic pigments responses of two varieties of Catharanthus roseus to triadimefon treatment. Comp. Rend. Biol., 331: 272-277

Lal, A. and Edwards, G.E. ; 1996. Analysis of inhibition of photosynthesis under water stress in the C4 species Amaranthus cruentus and Zea mays: electron transport, $\mathrm{CO} 2$ fixation and carboxylation capacity. Functional Plant Biology, 23: $403-412$

Monneveux, P., This, D. et Belhassen, E. ; 1997. Amélioration génétique de la résistance à la sécheresse. In C. Riou, R. Bonhomme, P. Chassin, A. Neveu, F. Papy (eds) : L'eau et l'espace rural. Production végétale et qualité de l'eau, INRA Éditions, Paris, 122-142.

Monneveux, P. ; Sánchez, C. ; Beck, D. and Edmeades, G.O. ; 2006. Drought tolerance improvement in tropical maize source populations : evidence of progress. Crop Sci., 46: 180191

Morizet, J. ; Robelin, M. ; Baucher, G. ; 1984. Résultats de 18 années d'observations lysimétriques sous climat limagnais. II. Étude des relations entre l'eau et la production végétale. Agronomie, 4: 407-416.

Prabhudeva, T.V. ; Chalapathi, M.M. ; Thimmegowda, S. ; Devakhumar, N. ; Rao, G.G. and
Mallikarjuna, K. ; 1998. Soil moisture stress and drought susceptibility index in sunflower. Indian Agric., 42: 287-289

Radhouane, L. et Ben Salem, M. ; 1998. Effet d'une contrainte hydrique appliquée à différents stades de développement sur certains paramètres écophysiologiques chez deus variétés d'orge : Rihane et Martin. Annales de I'INRAT, 71: 119140

Razmjoo, K. ; Heydarizadeh, P. and Sabzalian, M.R. ; 2008. Effect of salinity and drought stresses on growth parameters and essential oil content of Matricaria chamomile. Int. J. Agric. Biol., 10: $451-454$

Reynolds, M. and Tuberosa, R. ; 2008. Translational research impacting on crop productivity in drought-prone environments. Curr. Opin. Plant Biol., 11:171-179.

Saccardy K, Cornic G, Brulfert J, Reyss A. 1996. Effect of drought stress on net $\mathrm{CO} 2$ uptake in Zea leaves. Planta, 199: 589-595.

Sadras, V.O. and Malory, S.P. ; 1996. Soil-water thresholds for the responses of leaf expansion and gas exchange: A review. Field Crops Res., 47: 253-266.

Seghatoleslam, M.J., Kafi, M. et Majidi, E. ; 2008. Effect of drought stress at different growth stages on yield and water use efficiency of five proso millet (Panicum milliaceum L.) genotypes. Pak. J. Bot., 40(4) : 1427-1432.

Siddiqi, E.H. ; Asraf, M. and Akram, N.A. ; 2007. Variation in Seed germination and seedling growth in some diverse lines of safflower (Carthamus tinctorius L.) under salt stress. Pak. J. Bot., 39 (6) : 1937-1944.

Tester, M. and Bacic, A. ; 2005. Abiotic stress tolerance in grasses. From model plants to crop plants. Plant Physiol., 137: 791-793.

Tuinstra, M. R. ; Grote, E.M. ; Goldsbrough, P.B. and Ejeta, G. ; 1997. Genetic analysis of postflowering drought tolerance and components of grain development in Sorghum bicolor (L.) Moench. Molecular Breeding, 3: 439- 448.

Yari, L. ; Aghaalikhani, M. ; Khazaei, F. ; 2010. Effect of Seed priming duration and temperature on seed germination behavior of bread wheat ( Triticum aestivum L.). J. of Agric. Biol. Sci.,5 (1): 258280. 